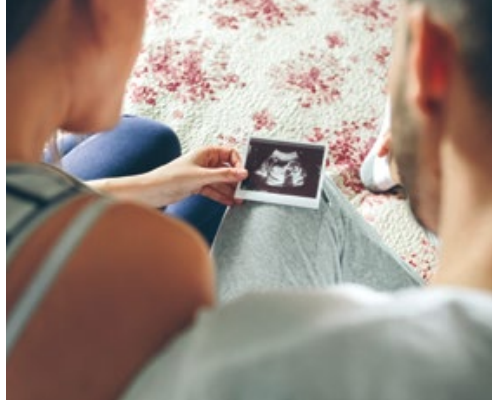

CPD

Alison Nankervis, Sarah Price, Jennifer Conn

\section{Background}

Gestational diabetes mellitus (GDM) is a common condition with risks for mother and baby. Type 2 diabetes mellitus (T2DM) and obesity are occurring with increasing frequency, and the incidence of GDM reflects this trend. Further, new diagnostic criteria have greatly increased the prevalence of GDM. Potential lifelong consequences exist for mother and baby if the condition goes untreated; however, adverse maternal and fetal effects can be ameliorated by effective pregnancy management.

\section{Objectives}

The aim of this article is to provide practical advice regarding GDM diagnosis, management and postpartum follow-up for general practitioners, who, increasingly, will be involved in GDM care.

\section{Discussion}

Controversy surrounds many aspects of GDM. Recommendations are frequently based on consensus. In the context of a deficient evidence base, any recommendations are open to debate. However, it is reasonable to suggest a pragmatic way forward, maintaining an open mind about the current diversity of practice and a preparedness to include further research findings into future practice.

\title{
Gestational diabetes mellitus
}

\author{
A pragmatic approach to diagnosis \\ and management
}

GESTATIONAL Diabetes Mellitus (GDM), or diabetes first recognised during pregnancy, is being diagnosed with increasing frequency. Women are entering pregnancy at an older age, are more likely to be obese or overweight and are often from a high-risk ethnic background. ${ }^{1}$ In addition, new diagnostic criteria, now widely adopted in Australia, ${ }^{2-4}$ have greatly increased diagnosis of GDM. In Australia, GDM now affects around 10\% of pregnancies but can occur in up to $30 \%$ in high-risk populations. ${ }^{5}$

GDM affects both mother and baby during pregnancy and in the long term. During pregnancy, women with GDM are at increased risk of pre-eclampsia, hypertension, early delivery, induction of labour and caesarean section. ${ }^{6}$ Long term, women with GDM have a greatly increased risk of developing type 2 diabetes mellitus (T2DM); Lee et al documented the cumulative risk to be $25.8 \%$ at 15 years post-pregnancy in a moderate-risk population. ${ }^{7}$ The cumulative incidence of T2DM is as high as $70 \%$ in some populations. ${ }^{8}$ The list of potential adverse effects in neonates is extensive and includes macrosomia, fetal death, shoulder dystocia, nerve palsy, hypoglycaemia and respiratory distress. ${ }^{6}$ Long-term effects of in utero exposure to hyperglycaemia, probably mediated by epigenetic mechanisms, ${ }^{9}$ relate to a propensity to overweight or obesity, dysglycaemia, dyslipidaemia and hypertension. These metabolic changes can occur as early as in the preschool years ${ }^{10}$ but are well established by adolescence in more than $50 \%$ of those whose mothers had GDM. ${ }^{11}$
In addition to strong evidence linking dysglycaemia in pregnancy with adverse outcomes, studies have shown the benefits of treating GDM. However, there are key questions yet to be answered. The first relates to the diagnosis of GDM. The association between adverse pregnancy outcomes and maternal glycaemia is a continuum, so current diagnostic criteria are based on consensus rather than definitive demarcation of risk. Second, the optimal timing and type of testing of women at high risk of GDM is uncertain, and the effects of early treatment unclear. Third, treatment targets for GDM remain the subject of ongoing debate. Finally, while there is agreement about the need for long-term follow-up after a GDM pregnancy, there are no consistent recommendations for its implementation.

How then can general practitioners (GPs) deal with these uncertainties, negotiate the difficult path between too much and too little medical intervention and provide optimal management for the women under their care?

\section{Diagnosis of GDM}

The recommendations for testing and diagnosis of GDM presented in this article were developed by the International Association of the Diabetes and Pregnancy Study Groups (IADPSG) and are based on the findings of the Hyperglycemia and Adverse Pregnancy Outcome (HAPO) study (Box 1$).^{6}$ This multinational observational study, involving more than 23,000 women, found a continuous increase in adverse pregnancy outcomes with increasing glycaemia. 
Consensus criteria for the diagnosis of GDM are based on a $75 \%$ increased risk of fetal weight $>90$ th percentile, fetal adiposity (body fat percentage) $>90$ th percentile, and cord blood C-peptide, reflecting increased fetal insulin production in response to maternal hyperglycaemia.

According to the IADPSG recommendations, diagnosis of GDM is made on a $75 \mathrm{~g}$ oral glucose tolerance test (OGTT) performed at 24-28 weeks gestation in all women not previously diagnosed with diabetes. The IADPSG diagnostic criteria for this time frame, ${ }^{2}$ shown in Box 1, may also provide a reasonable guide to glycaemic status between 16 weeks and full term. These criteria have been adopted by the Australasian Diabetes in Pregnancy

\section{Box 1. IADPSG diagnostic criteria for $\mathrm{GDM}^{2}$}

0-hour plasma glucose: $\geq 5.1 \mathrm{mmol} / \mathrm{L}$ 1-hour plasma glucose: $\geq 10.0 \mathrm{mmol} / \mathrm{L}$ 2-hour plasma glucose: $\geq 8.5 \mathrm{mmol} / \mathrm{L}$

Diagnosis is made on at least one elevated value. IADPSG, International Association of the Diabetes and Pregnancy Study Groups; GDM, gestational diabetes mellitus

Box 2. Risk factors for gestational diabetes mellitus from the Australasian Diabetes in Pregnancy Society guidelines $2014^{4}$

- Previous hyperglycaemia in pregnancy

- Previously elevated blood glucose level

- Maternal age $\geq 40$ years

- Ethnicity: Asian, Indian subcontinent, Aboriginal and Torres Strait Islander, Pacific Islander, Maori, Middle Eastern, non-white African

- Family history of diabetes mellitus (first-degree relative with diabetes or a sister with gestational diabetes mellitus)

- Pre-pregnancy body mass index $>30 \mathrm{~kg} / \mathrm{m}^{2}$

- Previous macrosomia (baby with birth weight $>4500 \mathrm{~g}$ or $>90$ th centile)

- Polycystic ovary syndrome

- Medications: corticosteroids, antipsychotics
Society ${ }^{4}$ and the Royal Australian and New Zealand College of Obstetricians and Gynaecologists, but are not universally accepted, ${ }^{12}$ as there is ongoing discussion about their validity.

\section{Testing for dysglycaemia in early pregnancy}

Women at high risk of developing GDM (Box 2) should be assessed clinically and tested early in pregnancy. At present, the major reason for early testing is to check for the confusingly named 'diabetes in pregnancy' or 'overt diabetes in pregnancy'.

\section{‘Diabetes in pregnancy'}

The diagnosis of 'diabetes in pregnancy' is made if World Health Organization (WHO) criteria for the diagnosis of diabetes outside of pregnancy are met. ${ }^{13}$ This identifies women who probably have pre-existing diabetes. The fetus is susceptible to complications of hyperglycaemia in early pregnancy, and the mother may have undiagnosed microvascular complications. Urgent management is required to normalise elevated glucose levels. The woman will then be treated throughout pregnancy as if she had pre-existing diabetes. If the clinical presentation appears atypical, the possibility that the woman has type 1 diabetes or a form of monogenic diabetes should be considered. Testing postpartum will clarify the diagnosis.

Options for early testing for 'diabetes in pregnancy' include OGTT, fasting plasma glucose, random plasma glucose and glycated haemoglobin (HbA1c) levels. The OGTT provides the most information but is often poorly tolerated in early pregnancy. $\mathrm{HbA} 1 \mathrm{c}$ is increasingly used but is not suitable for screening in women with thalassaemia or significant iron deficiency. When testing women on high-dose corticosteroids or metformin (usually for polycystic ovary syndrome), results should be interpreted cautiously.

Any one of the following values documented in the first trimester indicates likely 'diabetes in pregnancy':12
- OGTT

- Fasting plasma glucose: $\geq 7.0 \mathrm{mmol} / \mathrm{L}$

- 2-hour plasma glucose: $\geq 11.1 \mathrm{mmol} / \mathrm{L}$ - $\mathrm{HbA} 1 \mathrm{c} \geq 48 \mathrm{mmol} / \mathrm{mol}$ (6.5\%)

\section{Lesser degrees of dysglycaemia in pregnancy: 'Early GDM'}

While dysglycaemia in early pregnancy has been shown to be associated with a greater risk of adverse outcomes for both mother and fetus, ${ }^{14}$ the precise significance and consequences are not clear, nor have the risks or benefits of treatment been defined.

No test offers satisfactory alignment with a subsequent definitive diagnosis of GDM. ${ }^{15-17}$ Importantly, there are no tests that reliably predict pregnancy outcomes. Fasting glucose levels rise in early pregnancy, and GDM diagnosed on these levels is poorly replicated at routine 24-28 weeks' testing. ${ }^{18,19}$ A random plasma glucose level has low specificity, and the OGTT is not necessarily predictive of later GDM. HbA1c with levels of 41-46 mmol/mol (5.9-6.4\%) may identify women at higher risk of adverse pregnancy outcomes. ${ }^{20}$

Diagnosis and management of dysglycaemia early in pregnancy is not evidence-based. The following suggestions for further testing and management are intended for guidance only until robust evidence is available; alternatives could be considered equally valid (Box 3). Testing and management should always be considered in the context of the woman's clinical status. Addressing co-existent overweight and obesity through lifestyle advice may be helpful, given the association of these conditions with adverse pregnancy outcomes even in the absence of elevated blood glucose levels (BGLs). ${ }^{21}$ Local resources and the woman's own concerns are also factors in determining management.

\section{Management of GDM}

The keys to management of GDM are optimisation of lifestyle, self-monitoring of blood glucose (SMBG), intensive patient education and introduction of medication when warranted. ${ }^{22,23}$ 
Box 3. Approach to testing and management of dysglycaemia in early pregnancy

\section{Fasting plasma glucose}

5.1-5.9 mmol/L: Significance uncertain. Consider lifestyle advice. Oral glucose tolerance test (OGTT) at 16-18 weeks. 6.0-6.9 mmol/L: Closer association with subsequent formal diagnosis of gestational diabetes mellitus (GDM). Lifestyle advice. OGTT at 16-18 weeks, but discontinue test if fasting blood glucose elevated. Consider self-monitoring of blood glucose (SMBG).

\section{Random plasma glucose}

9-11 mmol/L: These levels are clearly elevated but their significance is not known. Warrants further testing - either fasting plasma glucose or $\mathrm{HbA1c}$, and act on the basis of these results.

\section{Glycated haemoglobin (HbA1c)}

5.9-6.4\%: These levels have reasonable sensitivity and specificity in predicting a formal diagnosis of GDM and have been associated with adverse pregnancy outcomes. Lifestyle advice. Consider SMBG if available, but it requires documented elevation of glucose levels for national diabetes services scheme registration.

\section{Lifestyle management}

The combination of optimal diet and appropriate exercise will result in $50-70 \%$ of women with GDM achieving management targets. Dietary principles involve meeting nutritional requirements for a healthy pregnancy, managing gestational weight gain, eliminating high glycaemic index (GI) carbohydrates and ensuring an adequate intake and distribution of low GI carbohydrates. ${ }^{24}$ Given the over-representation of certain ethnic groups with GDM, diets need to be reflective of usual diet and culture. Regular moderate exercise, such as walking, is helpful in maintaining fitness and can also assist greatly with maintaining glycaemic levels within target.

\section{Self-monitoring of blood glucose}

Effective monitoring is essential to GDM management. A plethora of blood glucose testing devices are readily available to health professionals, and many of these are supplied free of charge to women with GDM. Subsidised testing strips are available on completion of National Diabetes Service Scheme registration. A diabetes educator is invaluable in teaching women SMBG techniques and providing ongoing education and support. It is recommended that women initially measure BGLs four times per day: before breakfast and either one or two hours (depending on local practice) after the commencement of each meal.

\section{Treatment targets}

Target BGLs are an area of considerable debate, and at this time no evidence base supports specific targets. Fasting blood glucose targets vary from high 4 to $5.5 \mathrm{mmol} / \mathrm{L}$, depending on local guidelines.

The following values are published in the Australasian Diabetes in Pregnancy Society guidelines in the section 'for further research' and are followed by many, but by no means all, maternity centres: ${ }^{4,25}$

- Fasting capillary blood glucose: $\leq 5.0 \mathrm{mmol} / \mathrm{L}$

- 1-hour blood glucose: $\leq 7.4 \mathrm{mmol} / \mathrm{L}$

- 2-hour blood glucose: $\leq 6.7 \mathrm{mmol} / \mathrm{L}$ Further treatment is recommended if, after lifestyle intervention, values are exceeded on two or more occasions at a particular time point in one week.

\section{Pharmacological therapies}

Metformin, including extended-release formulations, has not been shown to harm mother, fetus or child; ${ }^{26-28}$ however, longterm effects are still being assessed and currently it is not widely used in Australia. Approximately $50 \%$ of women who commence metformin obtain satisfactory glycaemic control. ${ }^{29}$ However, for those women who ultimately require insulin, the use of metformin can result in a delay in achieving optimal glycaemia. If BGLs are substantially elevated, it is preferable to promptly institute insulin therapy, and if BGLs are elevated consistently at a particular time point, targeted insulin therapy is likely to be more effective than metformin. The use of metformin is contraindicated in the context of preeclampsia or fetal growth restriction.

Sulfonylureas, while used in a number of overseas centres, are not commonly prescribed for GDM in Australia. Sulfonylureas have been associated with higher birth weight and higher rates of neonatal hypoglycaemia. ${ }^{30,31}$ Because sulfonylureas cross the placenta, there are concerns about the potential effects of an insulin secretagogue on the fetal $\beta$-cell.

No other form of oral or injectable antihyperglycaemic therapy has documented safety in pregnancy.

\section{Insulin}

Insulin treatment in GDM is safe and effective and is usually the first choice of pharmacotherapy for GDM. ${ }^{31,32} \mathrm{Few}$ women are unable to manage insulin injections. In over 30 years at our centre, we have encountered no serious episodes of hypoglycaemia. Women using insulin sensibly and flexibly almost always achieve excellent glycaemic control. The use of a modified multidose regimen enables the use of insulin to match an individual woman's glycaemic profile.

In cases of fasting hyperglycaemia, medium-acting or long-acting insulin can be used at bedtime. The starting dose will depend on the degree of hyperglycaemia and the weight of the woman, but common starting doses are 4-8 units. The dose should be adjusted every two to three days to achieve target BGLs. In our institution we aim for BGLs consistently and clearly below $5 \mathrm{mmol} / \mathrm{L}$ but avoid BGLs below $4 \mathrm{mmol} / \mathrm{L}$.

For postprandial hyperglycaemia, after appropriate dietary education and review, introduce a short-acting insulin analogue before the affected meal(s). Starting doses are generally $4-8$ units. Adjust doses every two to three days to optimise BGLs at around 5-6 mmol/L. Twice daily pre-mixed insulin can be used if this is local practice, or if there is no particular pattern to the hyperglycaemia or if the woman is reluctant or unable to cope with more intensive therapy.

\section{Fetal monitoring, delivery and lactation}

Identification of the fetus at risk is important in GDM. Most GDM pregnancies, even when insulin treatment is required, are low risk. However, a small 
number of GDM pregnancies are deemed high risk and additional medical care is required. Careful clinical monitoring and the use of ultrasonography to document fetal size and wellbeing are the mainstays of management. A growth scan at 28-32 weeks can be useful in documenting macrosomia or growth restriction. A macrosomic fetus is more prone to complications during and after delivery, and warrants intensified monitoring and careful consideration of the type and timing of delivery. ${ }^{33}$

In most women, vaginal delivery at term is anticipated unless obstetric indications suggest a different approach. When steroids are indicated for early elective delivery, careful diabetes management is required. This may involve the introduction of insulin or insulin dose escalation, with the goal of maintaining euglycaemia. Pharmacotherapy should cease postpartum. The neonate, especially if macrosomic, is prone to hypoglycaemia, and all babies of GDM pregnancies need to have their blood glucose tested, ideally at around 1-2 hours after birth.

Women with GDM are encouraged to breastfeed. Some women may choose antenatal expressing to enable collection of colostrum for early neonatal feeding in the case of hypoglycaemia. ${ }^{34} \mathrm{~A}$ recent study has documented its safety from 36 weeks. ${ }^{34}$

\section{Postpartum testing and long-term follow-up}

It is important that all women who have had GDM have an OGTT to clearly document their glycaemic status after pregnancy, using WHO criteria for the diagnosis of diabetes. ${ }^{35}$ This testing is organised by either the maternity service or the woman's GP, ideally six weeks to three months postpartum. Type 1 diabetes or monogenic diabetes may present for the first time during pregnancy, although this is uncommon. Unusual clinical or biochemical characteristics or strong family history should prompt further investigation.

The timing and form of long-term follow-up is another area of controversy.
Annual measurement of $\mathrm{HbA} 1 \mathrm{c}$ is simple and practical and is reimbursed for diagnostic purposes in highrisk individuals. Another common recommendation is a fasting blood glucose measurement every 1-2 years, with the interval dependent on the woman's level of risk. ${ }^{4}$ Ideally, subsequent pregnancies will be planned, enabling optimisation of pregnancy preparation and early testing for hyperglycaemia.

\section{Key points}

- Before pregnancy:

- Identify risk of diabetes and, if high, suggest follow-up with $\mathrm{HbA} 1 \mathrm{c}$ testing.

- Provide general lifestyle advice, including the importance of optimising weight and managing overweight and obesity. Management of obesity before, during and after pregnancy is a critical issue in women of childbearing age.

- Where applicable, discuss smoking cessation and alcohol avoidance.

- Recommend routine laboratory tests.

- Commence folate supplementation at least one month before pregnancy.

- Early pregnancy:

- Test for 'diabetes in pregnancy' or dysglycaemia, according to risk profile.

- Provide general advice about gestational weight gain and healthy lifestyle.

- Commence insulin treatment if required or refer for specialist advice. Urgent referral is necessary for overt diabetes.

- During pregnancy:

- Test for GDM and provide appropriate management. If in rural or remote practice, consider telemedicine options or shared care.

- Postpartum:

- Organise OGTT and appropriate follow-up.

- Long term:

- Provide ongoing surveillance for diabetes and metabolic risk factors.

- Facilitate healthy lifestyle for mother and baby.

\section{Conclusion}

Diagnosis and effective management of GDM improve maternal and fetal outcomes. Early diagnosis of 'diabetes in pregnancy' enables prompt evaluation and treatment. Pragmatically, although controversial, $\mathrm{HbA1c}$ will have an increasing role in the recognition of diabetes in early pregnancy, as well as in long-term follow-up of women with previous GDM.

\section{Authors}

Alison Nankervis MBBS, MD, FRACP, Clinical Head, Diabetes, Royal Melbourne Hospital; Senior Physician, Diabetes and Endocrine Service, Royal Women's Hospital; Clinical Associate Professor, The University of Melbourne, Melbourne.

Alison.nankervis@mh.org.au

Sarah Price MBBS, DipObs, FRACP, Endocrinologist,

Royal Melbourne Hospital; Relieving Physician, Diabetes and Endocrine Service, Royal Women's Hospital; PhD candidate, The University of Melbourne, Melbourne

Jennifer Conn MBBS, M Clin Ed, FRACP,

Endocrinologist, Royal Melbourne Hospital; Physician, Diabetes and Endocrine Service, Royal Women's Hospital; Clinical Associate Professor, The University of Melbourne, Melbourne

Competing interests: None.

Provenance and peer review: Commissioned, externally peer reviewed.

\section{References}

1. Collier A, Abraham EC, Armstrong J, Godwin J, Monteath K, Lindsay R. Reported prevalence of gestational diabetes in Scotland: The relationship with obesity, age, socioeconomic status, smoking and macrosomia, and how many are we missing? J Diabetes Investig 2017;8(2):161-67. doi: 10.1111/ jdi.12552.

2. International Association of Diabetes and Pregnancy Study Groups Consensus Panel, Metzger BE, Gabbe SG, et al. International association of diabetes and pregnancy study groups recommendations on the diagnosis and classification of hyperglycemia in pregnancy. Diabetes Care 2010;33(3):676-82. doi: 10.2337/ dc09-1848.

3. Diagnostic criteria and classification of hyperglycaemia first detected in pregnancy: A World Health Organization Guideline. Diabetes Res Clin Pract 2014;103(3):341-63.

4. Nankervis A, Mclntyre HD, Moses R, et al. ADIPS consensus guidelines for the testing and diagnosis of gestational diabetes mellitus in Australia. NSW: Australasian Diabetes in Pregnancy Society, 2014.

5. Moses RG, Morris GJ, Petocz P, San Gil F, Garg D. The impact of potential new diagnostic criteria on the prevalence of gestational diabetes mellitus in Australia. Med J Aust 2011;194(7):338-40.

6. HAPO Study Cooperative Research Group, Metzger BE, Lowe LP, et al. Hyperglycemia and adverse pregnancy outcomes. N Engl J Med 2008;358(19):1991-2002. doi: 10.1056/ NEJMoa0707943. 
7. Lee AJ, Hiscock RJ, Wein P, Walker SP, Permezel M. Gestational diabetes mellitus: Clinical predictors and long-term risk of developing type 2 diabetes: $A$ retrospective cohort study using survival analysis. Diabetes Care 2007;30(4):878-83.

8. Song C, Lyu Y, Li C, et al. Long-term risk of diabetes in women at varying durations after gestational diabetes: A systematic review and meta-analysis with more than 2 million women. Obes Rev 2018;19(3):421-29. doi: 10.1111/obr.12645.

9. Ruchat SM, Hivert MF, Bouchard L. Epigenetic programming of obesity and diabetes by in utero exposure to gestational diabetes mellitus. Nutr Rev 2013;71 Suppl 1:S88-94. doi: 10.1111/nure.12057.

10. Boney CM, Verma A, Tucker R, Vohr BR. Metabolic syndrome in childhood: Association with birth weight, maternal obesity, and gestational diabetes mellitus. Pediatrics 2005;115(3):e290-96.

11. Clausen TD, Mathiesen ER, Hansen T, et al. High prevalence of type 2 diabetes and pre-diabetes in adult offspring of women with gestational diabetes mellitus or type 1 diabetes: The role of intrauterine hyperglycemia. Diabetes Care 2008;31(2):340-46.

12. Agarwal MM. Gestational diabetes mellitus: An update on the current international diagnostic criteria. World J Diabetes 2015;6(6):782-91. doi: 10.4239/wjd.v6.i6.782.

13. Wong T, Ross GP, Jalaludin BB, Flack JR. The clinical significance of overt diabetes in pregnancy. Diabet Med 2013;30(4):468-74. doi: 10.1111/ dme.12110.

14. Sweeting AN, Ross GP, Hyett J, et al. Gestational diabetes mellitus in early pregnancy: Evidence for poor pregnancy outcomes despite treatment. Diabetes Care 2016;39(1):75-81. doi: 10.2337/dc150433.

15. Hernandez TL, Friedman JE, Van Pelt RE, Barbour LA. Patterns of glycemia in normal pregnancy: Should the current therapeutic targets be challenged? Diabetes Care 2011;34(7):1660-68. doi: 10.2337/dc11-0241.

16. Mclntyre HD, Sacks DA, Barbour LA, et al. Issues with the diagnosis and classification of hyperglycemia in early pregnancy. Diabetes Care 2016;39(1):53-54. doi: 10.2337/dc15-1887.

17. Agarwal MM, Dhatt GS, Punnose J, Zayed R. Gestational diabetes: Fasting and postprandial glucose as first prenatal screening tests in a high-risk population. J Reprod Med 2007;52(4):299-305.

18. Cosson E, Carbillon L, Valensi P. High fasting plasma glucose during early pregnancy: A review about early gestational diabetes mellitus. J Diabetes Res 2017;2017:8921712. doi: 10.1155/2017/8921712.

19. Zhu WW, Yang HX, Wei YM, et al. Evaluation of the value of fasting plasma glucose in the first prenatal visit to diagnose gestational diabetes mellitus in China. Diabetes Care 2013;36(3):58690. doi: 10.2337/dc12-1157.

20. Hughes RC, Moore MP, Gullam JE, Mohamed $\mathrm{K}$, Rowan J. An early pregnancy $\mathrm{HbA} 1 \mathrm{c} \geq 5.9 \%$ $(41 \mathrm{mmol} / \mathrm{mol})$ is optimal for detecting diabetes and identifies women at increased risk of adverse pregnancy outcomes. Diabetes Care 2014;37(11):2953-59. doi: 10.2337/dc14-1312.

21. Mission JF, Marshall NE, Caughey AB. Pregnancy risks associated with obesity. Obstet Gynecol Clin North Am 2015;42(2):335-53. doi: 10.1016/j. ogc.2015.01.008
22. Landon MB, Spong CY, Thom E, et al. A multicenter, randomized trial of treatment for mild gestational diabetes. N Engl J Med 2009;361(14):1339-48. doi: 10.1056/ NEJMoa0902430.

23. Crowther CA, Hiller JE, Moss JR, et al. Effect of treatment of gestational diabetes mellitus on pregnancy outcomes. N Engl J Med 2005;352(24):2477-86.

24. Lee-Parritz A. Contemporary management of gestational diabetes. Curr Opin Endocrinol Diabetes Obes 2011;18(6):395-400. doi: 10.1097/ MED.0b013e32834cd3c4

25. Caissutti C, Saccone G, Ciardulli A, Berghella V. Very tight versus tight control: What should be the criteria for pharmacologic therapy dose adjustment in diabetes in pregnancy? Evidence from randomized controlled trials. Acta Obstet Gynecol Scand 2018;97(3):235-47. doi: 10.1111/ aogs.13257.

26. Niromanesh S, Alavi A, Sharbaf FR, Amjadi N, Moosavi S, Akbari S. Metformin compared with insulin in the management of gestational diabetes mellitus: A randomized clinical trial. Diabetes Res Clin Pract 2012;98(3):422-29. doi: 10.1016/j. diabres.2012.09.031.

27. McGrath RT, Glastras SJ, Scott ES, Hocking SL, Fulcher GR. Outcomes for women with gestational diabetes treated with metformin: A retrospective, case-control study. J Clin Med 2018;7(3). doi: 10.3390/jcm7030050.

28. Wouldes TA, Battin M, Coat S, Rush EC, Hague WM, Rowan JA. Neurodevelopmental outcome at 2 years in offspring of women randomised to metformin or insulin treatment for gestational diabetes. Arch Dis Child Fetal Neonatal Ed 2016. [Epub ahead of print] doi: 10.1136/ archdischild-2015-309602.

29. Rowan JA, Hague WM, Gao W, Battin MR, Moore MP; MiG Trial Investigators. Metformin versus insulin for the treatment of gestational diabetes. N Engl J Med 2008;358(19):2003-15. doi: 10.1056/ NEJMoa0707193.

30. Nachum Z, Zafran N, Salim R, et al. Glyburide versus metformin and their combination for the treatment of gestational diabetes mellitus: A randomized controlled study. Diabetes Care 2017;40(3):332-37. doi: 10.2337/dc16-2307.

31. Balsells M, García-Patterson A, Solà I, Roqué M, Gich I, Corcoy R. Glibenclamide, metformin, and insulin for the treatment of gestational diabetes: A systematic review and meta-analysis. BMJ 2015;350:h102. doi: 10.1136/bmj.h102.

32. Brown J, Grzeskowiak L, Williamson K, Downie $M R$, Crowther CA. Insulin for the treatment of women with gestational diabetes. Cochrane Database Syst Rev 2017;11:CD012037. doi: 10.1002/14651858.CD012037.pub2.

33. Maso G, Piccoli M, Parolin S, Restaino S, Alberico S. Diabetes in pregnancy: Timing and mode of delivery. Curr Diab Rep 2014;14(7):506. doi: 10.1007/s11892-014-0506-0.

34. Forster DA, Moorhead AM, Jacobs SE, et al. Advising women with diabetes in pregnancy to express breastmilk in late pregnancy (Diabetes and Antenatal Milk Expressing [DAME]): A multicentre, unblinded, randomised controlled trial. Lancet 2017;389(10085):2204-13. doi: 10.1016/S0140-6736(17)31373-9.

35. Kitzmiller JL, Dang-Kilduff L, Taslimi MM. Gestational diabetes after delivery. Short-term management and long-term risks. Diabetes Care 2007;30 Suppl 2:S225-35. doi: 10.2337/dc07-s221. 